\title{
KDM6A suppresses hepatocellular carcinoma cell proliferation by negatively regulating the TGF-ß/SMAD signaling pathway
}

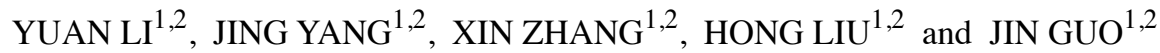 \\ ${ }^{1}$ Department of General Surgery, Gansu Provincial Hospital; ${ }^{2}$ Clinical Center of General Surgery, \\ People's Clinical Medical College of Lanzhou University, Lanzhou, Gansu 730000, P.R. China
}

Received December 13, 2019; Accepted May 22, 2020

DOI: $10.3892 /$ etm.2020.9000

\begin{abstract}
Lysine demethylase 6A (KDM6A) is a Jumonji-C domain-containing histone demethylase that specifically catalyzes the removal of histone $\mathrm{H} 3$ lysine-27 trimethylation. KDM6A is a member of the KDM6 family, the biological role of which has been reported in various types of cancer, including bladder and lung cancer, as well as pancreatic ductal adenocarcinoma. However, the role of KDM6A in hepatocellular carcinoma (HCC) is not completely understood. Therefore, the present study aimed to determine the biological function of KDM6A in HCC progression. The expression profile of KDM6A was examined in HCC surgical specimens using reverse transcription-quantitative PCR. In addition, the role of KDM6A in the proliferation capacities of HCC cell lines was examined in vitro and in vivo using crystal violet and MTT assays. The underlying mechanism by which KDM6A exerts its function was explored by western blotting. The present study indicated that KDM6A was significantly downregulated in HCC tissues compared with normal control tissues. The role of KDM6A in HCC cell proliferation was also determined. KDM6A overexpression significantly inhibited HCC cell proliferation, whereas KDM6A knockdown significantly promoted HCC cell proliferation compared with the corresponding control groups. Consistently, KDM6A overexpression suppressed HCC cell tumorigenesis in vivo. The western blotting results indicated that KDM6A overexpression decreased the phosphorylation levels of $\operatorname{smad} 2$, whereas KDM6A knockdown increased the phosphorylation levels of $\operatorname{smad} 2$ compared with the corresponding control groups. Therefore, the present study suggested that KDM6A may inhibit HCC cell proliferation by negatively regulating the TGF- $\beta /$ SMAD signaling pathway, suggesting that KDM6A may serve as a potential target for the diagnosis and treatment of HCC.
\end{abstract}

Correspondence to: Dr Jin Guo, Department of General Surgery, Gansu Provincial Hospital, 204 Donggangxi Road, Lanzhou, Gansu 730000, P.R. China

E-mail: guojin2019222@sina.com

Key words: lysine demethylase 6A, proliferation, molecular target, hepatocellular carcinoma, transforming growth factor- $\beta / \mathrm{SMAD}$

\section{Introduction}

Hepatocellular carcinoma (HCC) is one of the most common malignancies worldwide, resulting in high morbidity and mortality, with 854,000 new cases and 810,000 cases of mortality each year (1). HCC is also the fifth most commonly occurring cancer, as well as the third leading cause of cancer-related death worldwide (2). Multiple therapeutic strategies, such as liver resection, transarterial chemoembolization, radiotherapy and sorafenib administration, have been used for the treatment of patients with HCC. However, the prognosis of patients with HCC remains poor due to its complexity, high recurrence and early vascular invasion $(3,4)$. Increasing evidence has revealed that HCC is associated with multi-gene mutations (5). Molecular targeting has been suggested as a novel therapeutic approach for patients with advanced HCC, as it significantly increases the survival time of patients and has favorable curative effects (6). Therefore, understanding the development of HCC and identifying novel molecular therapeutic targets has received increasing attention.

Lysine demethylase 6A (KDM6A; also known as ubiquitously transcribed $\mathrm{X}$ chromosome tetratricopeptide repeat protein) is a Jumonji-C domain-containing histone demethylase that catalyzes the removal of histone $\mathrm{H} 3$ lysine-27 trimethylation $(7,8)$. As a member of the KDM6 family, KDM6A is also a key molecule that regulates cell fate decisions and cell identity during normal development by controlling the expression of pluripotency and lineage-specific genes $(9,10)$. It has also been reported that KDM6A is frequently targeted by somatic inactivating mutations in various types of cancer, such as esophageal and urethral cancer, as well as pancreatic ductal adenocarcinoma and multiple myeloma (11). Mechanistically, KDM6A mutations suppress cell proliferation by activating retinoblastoma protein-related genes, which suggests that KDM6A serves as a putative tumor suppressor (12). Furthermore, the biological function of KDM6A in a number of malignancies, including bladder and lung cancer, as well as pancreatic ductal adenocarcinoma, has been reported (13-16). However, the cellular and molecular mechanisms underlying KDM6A in HCC are not completely understood.

The present study investigated the importance of KDM6A during HCC progression. The results indicated that KDM6A expression was downregulated in human $\mathrm{HCC}$ tissues compared with corresponding normal control tissues. 
In addition, KDM6A overexpression inhibited HCC cell proliferation in vitro and in vivo. Therefore, the present study identified a potential molecular mechanism underlying the role of KDM6A during HCC development.

\section{Materials and methods}

HCC tissue samples. A total of 30 paired primary HCC tissues and adjacent normal tissues(distance, $<2 \mathrm{~cm}$ ) were obtained from patients (age, 32-68 years; mean age, 46 \pm 3.2 years; 22 male patients and 8 female patients) who had undergone a hepatectomy between February and July 2019 at Gansu Provincial Hospital (Lanzhou, China). The inclusion criteria were as follows: i) Patients clinically diagnosed with liver cancer following surgery; ii) R0 resection based on histological examinations; and iii) paired normal tissue was adjacent to tumor tissue (distance, $<2 \mathrm{~cm}$ ). The exclusion criteria were as follows: i) Patients with distant metastasis; and ii) patients who had received radiotherapy or chemotherapy before surgery. Paired samples were subjected to RNA extraction for reverse transcription-quantitative PCR (RT-qPCR) and immunohistochemistry (IHC) analyses. The present study was approved by the Institutional Review Board of The Institute for Gansu Provincial Hospital (Lanzhou, China). All patients provided written informed consent.

$I H C$. Tumor tissues and paired normal tissues were fixed in $4 \%$ formalin at room temperature for $24 \mathrm{~h}$, embedded in paraffin and cut into $5-\mu \mathrm{m}$ thick consecutive sections. Paraffin-embedded tissue sections were deparaffinized in xylene, rehydrated using a descending ethanol gradient before being subjected to antigen retrieval in a sodium citrate solution ( $\mathrm{pH} \mathrm{6.0)}$ ) for $20 \mathrm{~min}$ at $98^{\circ} \mathrm{C}$ and endogenous peroxidase activity blocking with $3 \%$ hydrogen peroxide. The sections were washed three times with $0.01 \mathrm{M}$ PBS for $5 \mathrm{~min}$ each and blocked in $0.01 \mathrm{M}$ PBS containing $0.3 \%$ Triton X-100 and 5\% BSA (Gibco; Thermo Fisher Scientific, Inc.) at room temperature for $1 \mathrm{~h}$. Subsequently, sections were incubated with an anti-KDM6A antibody (cat. no. orb333886; 1:100; Biorbyt Ltd.) at $4^{\circ} \mathrm{C}$ overnight. After washing with $0.01 \mathrm{M}$ PBS, sections were incubated with an anti-rabbit horseradish peroxidase-conjugated secondary antibody (cat. no. 7074; 1:5,000; Cell Signaling Technology, Inc.) at room temperature for $1 \mathrm{~h}$. Subsequently, DAB substrate was used to visualize KDM6A protein expression levels. Stained sections were observed in five randomly selected, independent high-power microscopic field of view using an inverted light microscope (magnification, $\mathrm{x} 400$ ).

$R N A$ extraction and $R T-q P C R$. Tissues were snap-frozen in liquid nitrogen and stored at $-80^{\circ} \mathrm{C}$ until further analysis. Total RNA was extracted from tumor tissues and adjacent noncancerous tissues using TRIzol ${ }^{\circledR}$ reagent (Invitrogen; Thermo Fisher Scientific, Inc.). RNA (2 $\mu \mathrm{g})$ was reverse transcribed into cDNA using the PrimeScript ${ }^{\mathrm{TM}}$ RT Reagent kit (Takara Bio, Inc.), according to the manufacturer's instructions, with the following thermocycling conditions: $37^{\circ} \mathrm{C}$ for $15 \mathrm{~min}$ and $85^{\circ} \mathrm{C}$ for $5 \mathrm{sec}$. Subsequently, qPCR was performed using SYBR ${ }^{\circledR}$ Premix Ex Taq (Takara Bio, Inc.). The sequences of the primers used for qPCR are listed in
Table I. The following thermocycling conditions were used for qPCR: Initial denaturation for $1 \mathrm{~min}$ at $95^{\circ} \mathrm{C} ; 40$ cycles of denaturation for $20 \mathrm{sec}$ at $95^{\circ} \mathrm{C}$, annealing at $56^{\circ} \mathrm{C}$ for $60 \mathrm{sec}$ and extension at $72^{\circ} \mathrm{C}$ for $2 \mathrm{~min}$; followed by final extension at $72^{\circ} \mathrm{C}$ for $6 \mathrm{~min}$. mRNA expression levels were quantified using the $2^{-\Delta \Delta C q}$ method (17) and normalized to the internal reference gene GAPDH.

Cell culture. Human HCC cell lines (YY-8103, SNU-398, MHCC97-L, Hep3B, LM3 and Huh7) and 293T cells were purchased from The Cell Bank of Type Culture Collection of the Chinese Academy of Sciences. Cells were cultured in DMEM (Invitrogen; Thermo Fisher Scientific, Inc.) supplemented with $100 \mathrm{U} / \mathrm{ml}$ penicillin, $100 \mathrm{mg} / \mathrm{ml}$ streptomycin and $10 \%$ FBS (Gibco; Thermo Fisher Scientific, Inc.) at $37^{\circ} \mathrm{C}$ with $5 \% \mathrm{CO}_{2}$. The Huh7, LM3 and Hep3B cell lines have high invasive characteristics, whereas the YY-8103, SNU-398 and MHCC-97L cell lines have less invasive characteristics (18).

Cell transfection. A KDM6A coding sequence was constructed and inserted into the p23-3xflag-GFP vector (Invitrogen; Thermo Fisher Scientific, Inc.) to generate a KDM6A overexpression vector. The empty $\mathrm{p} 23$ vector was used as the negative control. Lentiviral short hairpin (sh)RNAs targeting KDM6A were designed and constructed into pLKO.1-TRC vectors (Shanghai GenePharma Co., Ltd.). A non-targeting scrambled (SCR) oligonucleotide (Shanghai GenePharma Co., Ltd.) constructed into pLKO.1-TRC vectors served as the negative control. The sequences of the shRNAs are provided in Table I.

To produce lentiviral particles for KDM6A overexpression and knockdown, the core plasmid $(1.5 \mu \mathrm{g})$ was co-transfected with the packaging plasmids $3.0 \mu \mathrm{g}$ pMD2.G and $3.0 \mu \mathrm{g}$ psPAX2 (Shanghai GenePharma Co., Ltd) into $293 \mathrm{~T}$ cells $\left(5 \times 10^{5}\right.$ cells per well) using a calcium phosphate co-precipitation method in six-well plates (19). At $12 \mathrm{~h}$ post-transfection, the medium was refreshed. At 24 and $48 \mathrm{~h}$ post-transfection, the cell culture supernatants containing the virus were collected and filtered through a $0.45 \mu \mathrm{m}$ membrane. Subsequently, the virus was concentrated by centrifugation at $50,000 \mathrm{x} \mathrm{g}$ for $140 \mathrm{~min}$ at $4^{\circ} \mathrm{C}$. The pellet was resuspended in DMEM solution, aliquoted and stored at $-80^{\circ} \mathrm{C}$ until further use. For the transduction process, Huh7 and LM3 cells $\left(1 \times 10^{6}\right.$ cells per well) were grown to $60 \%$ confluence in 6 -well plates and transduced with $40 \mu \mathrm{l}$ viral supernatant $\left(1 \times 10^{7}\right.$ units per well) and $5 \mu \mathrm{g} / \mathrm{ml}$ polybrene (Hanheng Biological Technology Co., Ltd.). After $24 \mathrm{~h}$ at $37^{\circ} \mathrm{C}$, the medium was refreshed and the cells were transferred to $10-\mathrm{cm}$ dishes. After 2 days at $37^{\circ} \mathrm{C}$, KDM6A-overexpression Huh7 and LM3 cells were screened by green fluorescence via flow cytometry using an IX-71 flow cytometer (Olympus Corporation). KDM6A-knockdown YY-8103 and SNU-398 cells were cultured and screened in medium containing $3 \mu \mathrm{g} / \mathrm{ml}$ puromycin (Hanheng Biological Technology Co., Ltd.) for 4 days at $37^{\circ} \mathrm{C}$. Subsequently, individual puromycin-resistant colonies were isolated and used for subsequent experiments. Transfection efficiency was verified by western blotting.

Western blotting. Total protein was extracted from cells using RIPA Lysis Buffer (Thermo Fisher Scientific, Inc.) supplemented with phenylmethylsulfonyl fluoride (Thermo Fisher 
Table I. Primer and shRNA sequences.

\begin{tabular}{|c|c|}
\hline Gene & Sequence $\left(5^{\prime} \rightarrow 3^{\prime}\right)$ \\
\hline KDM6A & $\begin{array}{l}\text { F: TTCCTCGGAAGGTGCTATTCA } \\
\text { R: GAGGCTGGTTGCAGGATTCA }\end{array}$ \\
\hline GAPDH & $\begin{array}{l}\text { F: ATGACCCCTTCATTGACCTCA } \\
\text { R: GAGATGATCACCCTTTTGGCT }\end{array}$ \\
\hline KDM6A-SCR & $\begin{array}{l}\text { F: TTCTCCGAACGTGTCACGT } \\
\text { R: ACGTGACACGTTCGGAGAA }\end{array}$ \\
\hline KDM6A-sh1 & $\begin{array}{l}\text { F: CCGGGCACATAGACTAAGGAATAAACTCGAGTTTATTCCTTAGTCTATGTGCTTTTTG } \\
\text { R: AATTCAAAAAGCACATAGACTAAGGAATAAACTCGAGTTTATTCCTTAGTCTATGTGC }\end{array}$ \\
\hline KDM6A-sh2 & $\begin{array}{l}\text { F: CCGGGCAGCACGAATTAAGTATTTACTCGAGTAAATACTTAATTCGTGCTGCTTTTTG } \\
\text { R: AATTCAAAAAGCAGCACGAATTAAGTATTTACTCGAGTAAATACTTAATTCGTGCTGC }\end{array}$ \\
\hline
\end{tabular}

sh, short hairpin RNA; KDM6A, lysine demethylase 6A; SCR, scrambled.

A

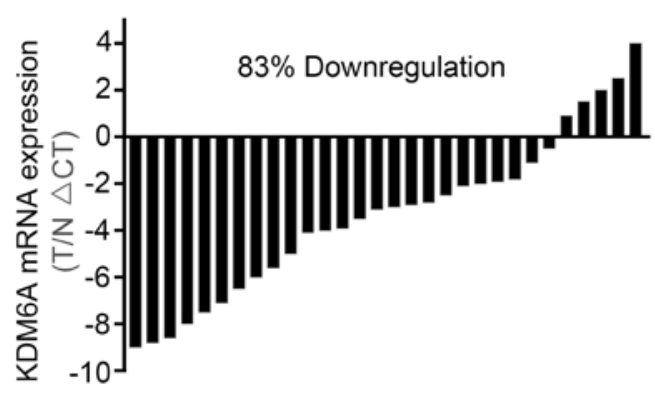

C

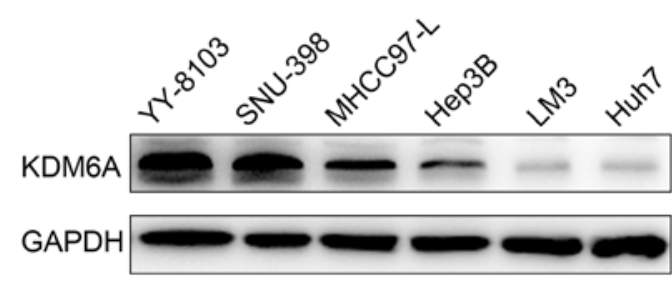

B

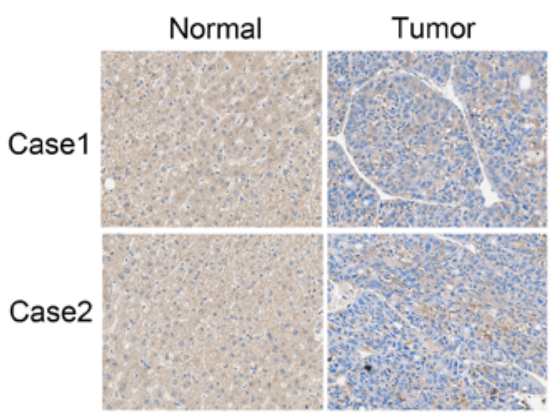

D

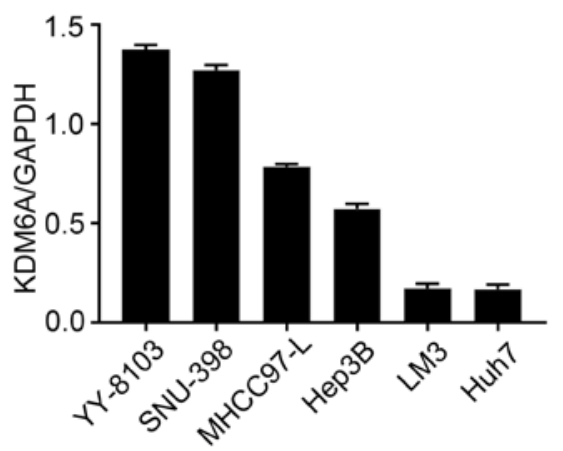

Figure 1. KDM6A expression is decreased in HCC clinical samples and cell lines. (A) KDM6A mRNA expression levels in 30 paired tumor and matched normal hepatic tissues. (B) KDM6A expression levels in tumor and matched normal hepatic tissue samples obtained from two patients as determined by immunohistochemistry. Magnification, x400. KDM6A protein expression levels in six HCC cell lines (YY-8103, SNU-398, MHCC97-L, LM3 and Huh7) were (C) determined by western blotting and (D) semi-quantified. KDM6A, lysine demethylase 6A; HCC, hepatocellular carcinoma.

Scientific, Inc.). Total cell lysates were centrifuged at $10,000 \mathrm{x} \mathrm{g}$ for $15 \mathrm{~min}$ at $4^{\circ} \mathrm{C}$, following which the supernatant was collected. Protein concentration of the supernatant was determined using Bradford reagent (Sigma-Aldrich; Merck KGaA). Protein ( $20 \mu \mathrm{g}$ per lane) was separated via 10\% SDS-PAGE and transferred onto nitrocellulose membranes (EMD Millipore). The membranes were blocked with $5 \%$ fat-free milk for $1 \mathrm{~h}$ at room temperature. Subsequently, the membranes were incubated at $4^{\circ} \mathrm{C}$ overnight with the following primary antibodies: Anti-KDM6A (1:1,000; cat. no. orb333886; Biorbyt Ltd.), anti-transforming growth factor (TGF)- $\beta(1: 1,000$; cat. no. 3711 ; Cell Signaling Technology, Inc.), anti-phosphorylated (p)-smad2 (1:1,000; cat. no. 18338; Cell Signaling Technology, Inc.), anti-Smad2 (1:1,000; cat. no. 12570-1-AP; ProteinTech Group, 
A
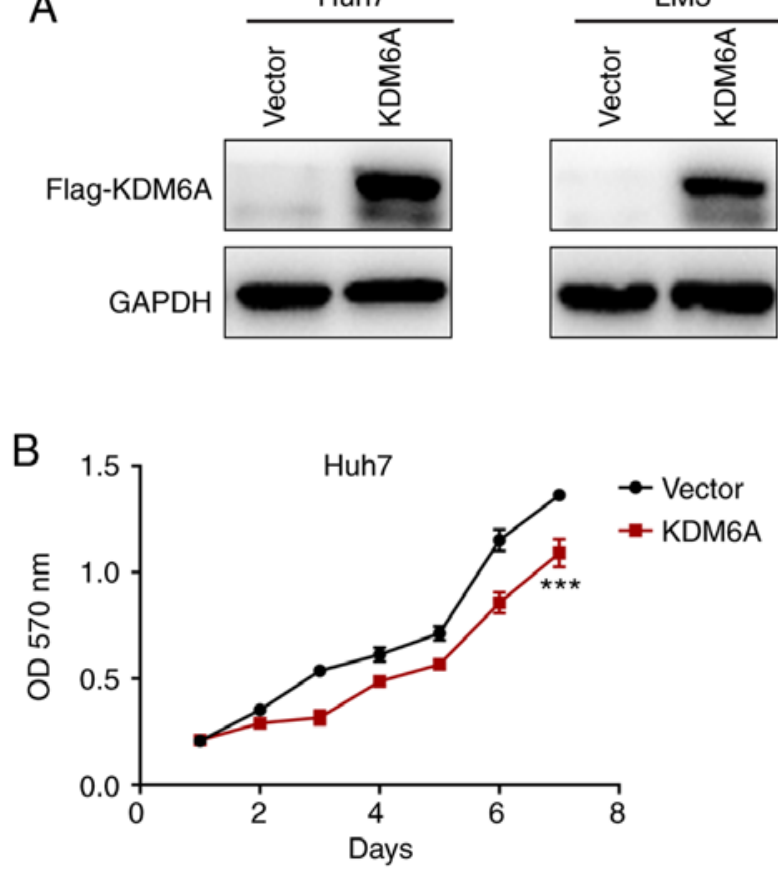

D

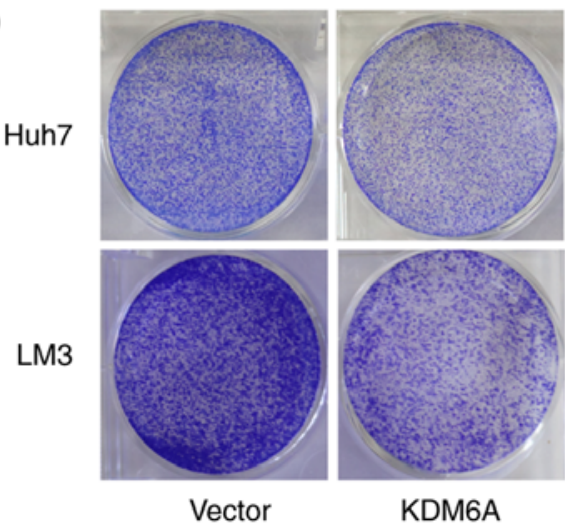

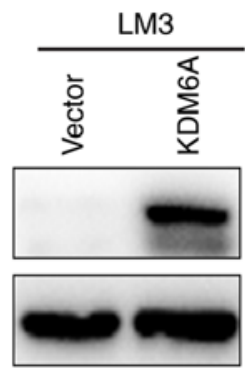
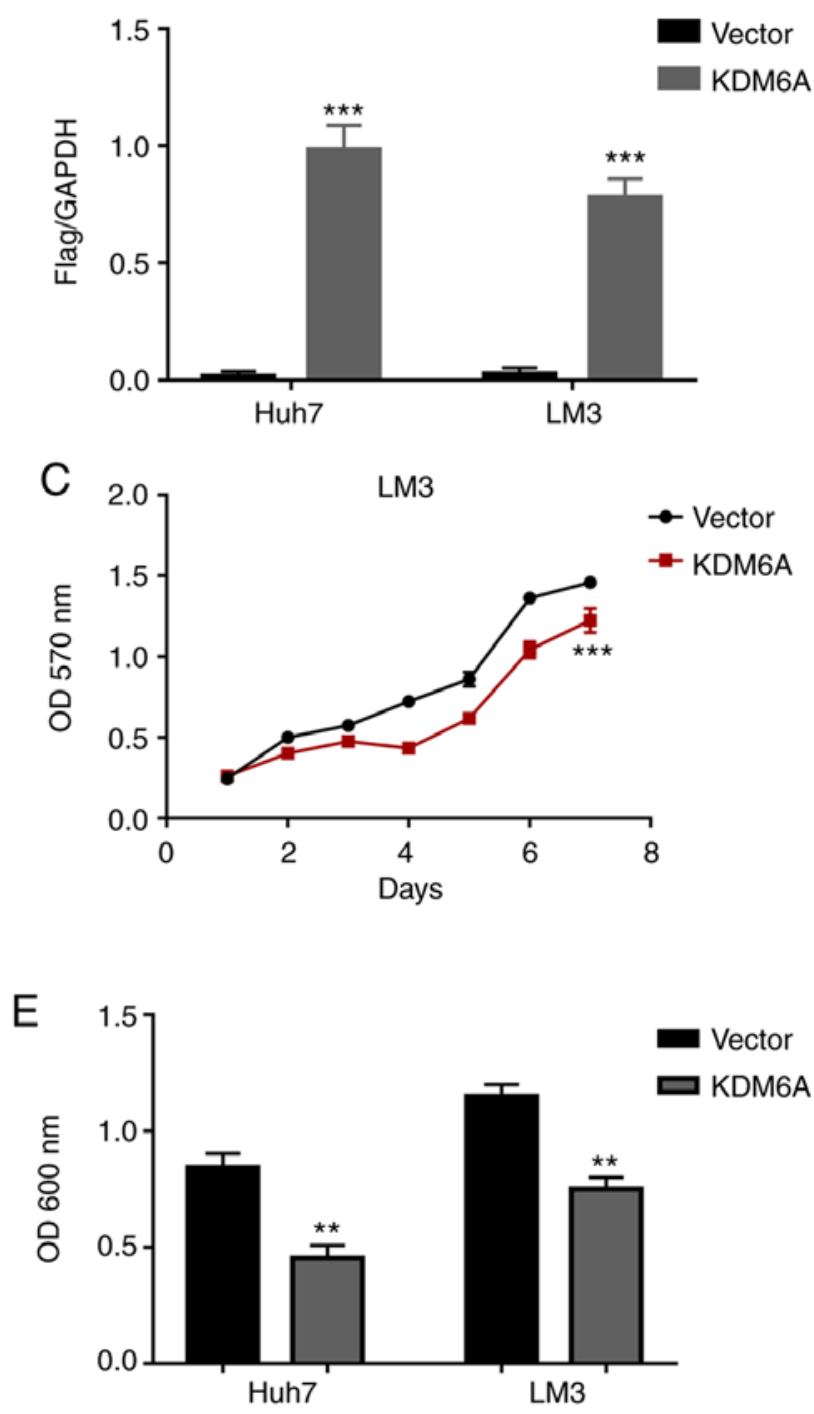

Figure 2. KDM6A overexpression inhibits hepatocellular carcinoma cell proliferation. (A) Transfection efficiency of KDM6A overexpression in Huh7 and LM3 cells. The effect of KDM6A overexpression on (B) Huh7 and (C) LM3 cell proliferation was measured by performing an MTT assay. (D) The effect of KDM6A overexpression on Huh7 and LM3 cell proliferation was detected by performing a crystal violet assay. Magnification, $\mathrm{x} 4$. (E) The OD value of the crystal violet assay in Huh7 and LM3 cells. ${ }^{* *} \mathrm{P}<0.01$ and ${ }^{* * *} \mathrm{P}<0.001$ vs. vector. KDM6A, lysine demethylase $6 \mathrm{~A}$; OD, optical density.

Inc.), anti-p-smad4 (1:1,000; cat. no. 10231-8-AP; ProteinTech Group, Inc.), anti-smad4 (1:1,000; cat. no. 10231-1-AP; ProteinTech Group, Inc.), anti-proliferating cell nuclear antigen (PCNA; 1:1,000; cat. no. 10205-2-AP; ProteinTech Group, Inc.), anti-Ki67 (1:1,000; cat. no. 27309-1-AP; ProteinTech Group, Inc.), anti-GAPDH (1:1,000; cat. no. 10494-1-AP; ProteinTech Group, Inc.) and anti-Flag (1:4,000; cat. no. F7425; Sigma-Aldrich; Merck KGaA). Following primary incubation, the membranes were incubated with an anti-rabbit $(1: 5,000$; cat. no. 7074; Cell Signaling Technology, Inc.) or anti-mouse (1:3,000; cat. no. 7076; Cell Signaling Technology, Inc.) horseradish peroxidase-conjugated secondary antibody for $1 \mathrm{~h}$ at room temperature. The immunoreactive protein bands were visualized using an enhanced chemiluminescence kit (Pierce; Thermo Fisher Scientific, Inc.) and a Gel Dox XR system (Bio-Rad Laboratories, Inc.). GAPDH was used as the loading control. ImageJ software (version 1.8.0, National Institutes of Health) was used for quantification of western blotting.
Crystal violet assay. Wild-type and transfected cells were seeded $\left(1 \times 10^{3}\right.$ cells/well) into 6 -well plates. Cells were cultured in DMEM (Invitrogen; Thermo Fisher Scientific, Inc.) supplemented with $10 \%$ FBS and the medium was changed every 3 days. After 2 weeks, the medium was removed and cells were fixed with $20 \%$ methanol at room temperature for $10 \mathrm{~min}$. Subsequently, cells were stained with $0.5 \%$ crystal violet at room temperature for $10 \mathrm{~min}$. Stained cells were washed to PBS and observed using a light microscope (magnification, $\mathrm{x} 4$ ). Subsequently, $1 \mathrm{ml}$ glacial acetic acid was added to each well and the optical density of each well was measured at a wavelength of $600 \mathrm{~nm}$ using a microplate reader.

MTT assay. Cells were seeded ( $1 \times 10^{3}$ cells/well) into 96-well plates in triplicate. After incubation for $1-7$ days at $37^{\circ} \mathrm{C}, 20 \mu \mathrm{l}$ MTT solution $(5 \mathrm{mg} / \mathrm{ml})$ was added to each well and incubated at $37^{\circ} \mathrm{C}$ for $4 \mathrm{~h}$. Subsequently, the culture medium was removed, $200 \mu \mathrm{l}$ DMSO was added and the plates were gently 
A
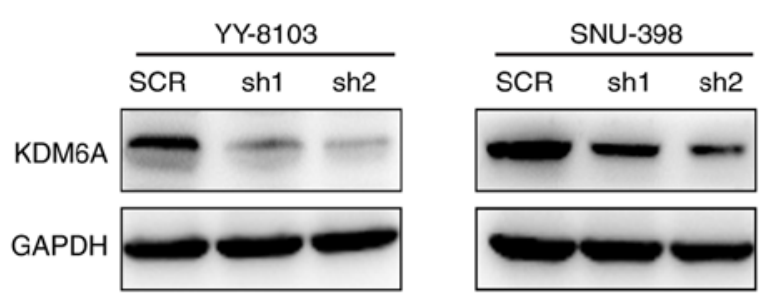

B

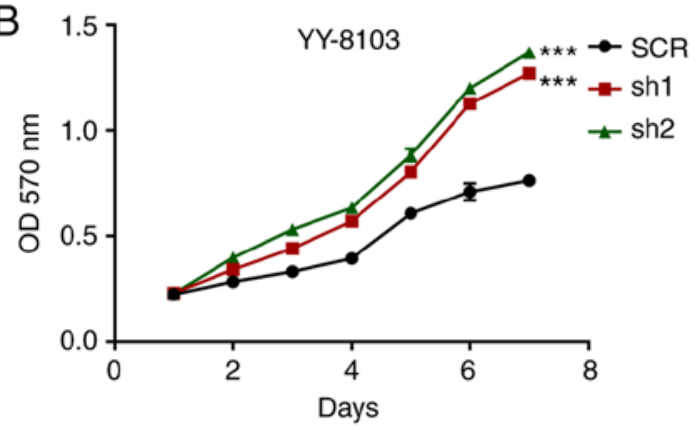

D

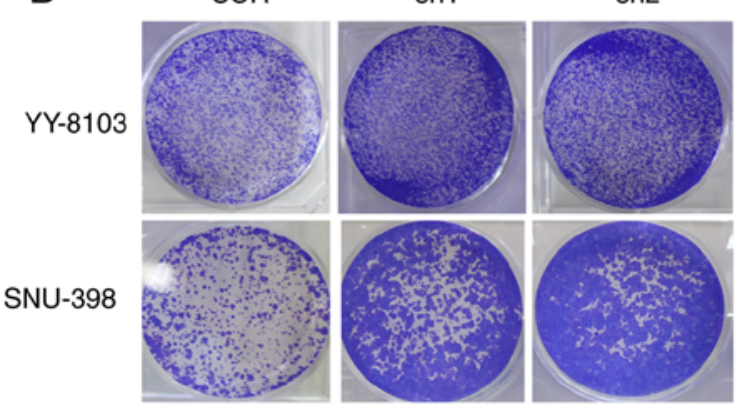

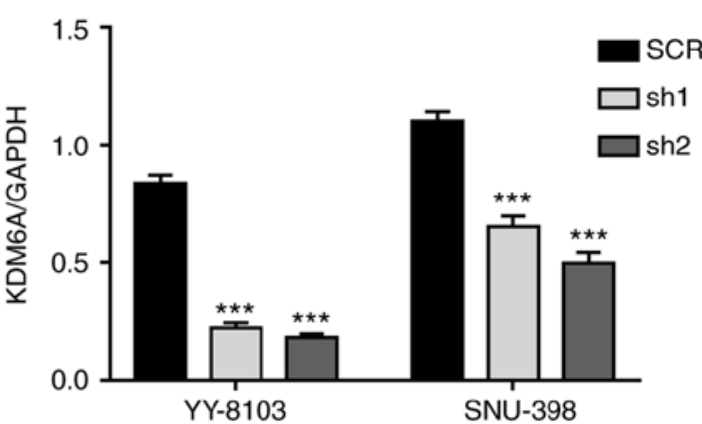

C

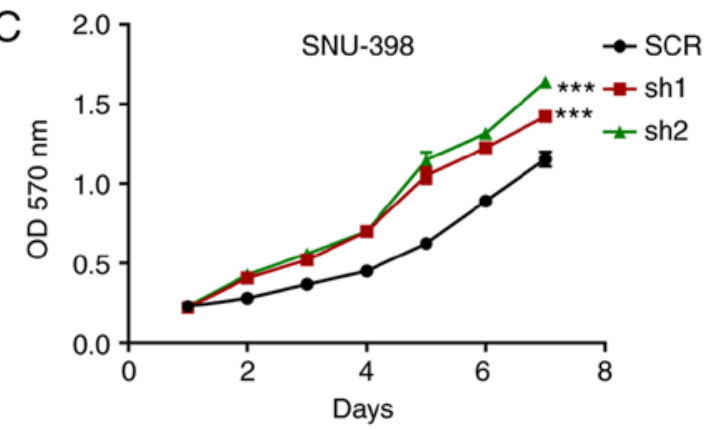

E

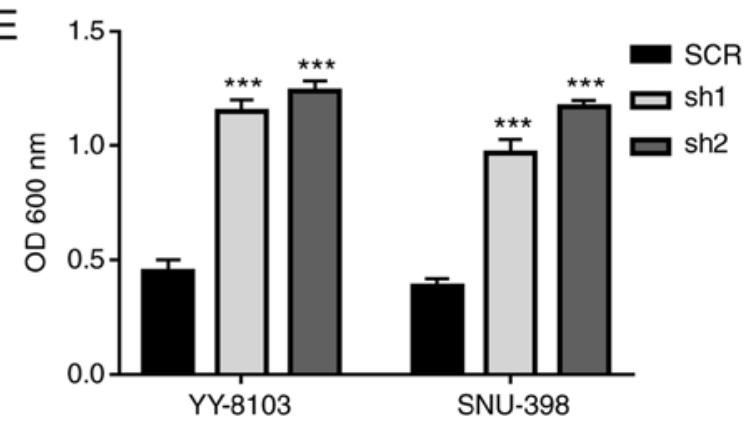

Figure 3. KDM6A knockdown promotes hepatocellular carcinoma cell proliferation. (A) Transfection efficiency of KDM6A knockdown in YY-8103 and SNU-398 cells. (B) The effect of KDM6A knockdown on (B) YY-8103 and (C) SNU-398 cell proliferation was measured by performing an MTT assay. (D) The effect of KDM6A knockdown on YY-8103 and SNU-398 cell proliferation was detected by performing a crystal violet assay. Magnification, $\mathrm{x} 4$. (E) The OD value of the crystal violet assay in YY-8103 and SNU-398 cells. ${ }^{* * *} \mathrm{P}<0.001$ vs. SCR. KDM6A, lysine demethylase 6 A; OD, optical density; SCR, scrambled; sh, short hairpin RNA.

agitated to dissolve the formazan crystals. The absorbance of each well was measured at a wavelength of $570 \mathrm{~nm}$ using an automatic microplate reader.

In vivo tumorigenesis analysis. A total of 8 male BALB/c nude mice (age, $\sim 5$ weeks; median weight, $20 \mathrm{~g}$; weight range, 18-21 g) were obtained from Beijing Huafukang Bioscience Co., Ltd. Mice were housed with free access to regular chow diet and water under specific pathogen-free conditions in laboratory cages at $23 \pm 3^{\circ} \mathrm{C}$ with $35 \pm 5 \%$ humidity and $12-\mathrm{h}$ light/dark cycles. The mice divided into were divided into two groups ( $\mathrm{n}=4$ per group): i) Huh7 p23 negative control Vector and ii) Huh7 KDM6A. KDM6A overexpression vector- or p23 negative control vector-transfected Huh7 cells $\left(1 \times 10^{6}\right.$ cells in a total volume of $100 \mu \mathrm{l}$ PBS) were then subcutaneously injected using a $1 \mathrm{ml}$ injection syringe into the right flank of each mouse in their corresponding groups. Tumor size was measured on a weekly basis. Tumor volume was calculated according to the following formula: Tumor volume $\left(\mathrm{mm}^{3}\right)=0.5 x$ lengthxwidth ${ }^{2}$. At the end of the experiment (week 4), mice were sacrificed by cervical dislocation. The tumors were excised, photographed and weighed. The maximum tumor volume observed was $609 \mathrm{~mm}^{3}$. All animal experiments were performed according to guidelines of the Institutional Animal Care and Use Committee of Gansu Provincial Hospital (20) and were approved by Ethics Committee of the Gansu Provincial Hospital.

Statistical analysis. Statistical analyses were performed using GraphPad Prism (version 5; GraphPad Software, Inc.). Data are presented as the mean \pm SD. Comparisons between two groups were determined using the unpaired Student's t-test. Comparisons between multiple groups were determined using one-way ANOVA followed Tukey's post hoc test. $\mathrm{P}<0.05$ was considered to indicate a statistically significant difference. All experiments were performed in triplicate.

\section{Results}

KDM6A is downregulated in HCC tissues. To explore the potential roles of KDM6A in HCC, the mRNA expression 
A

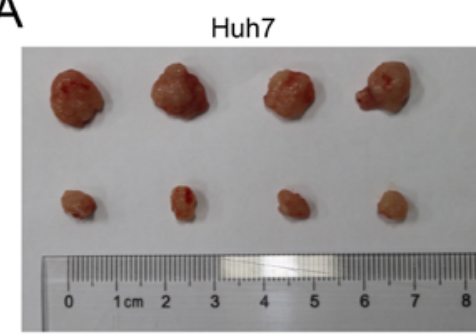

C

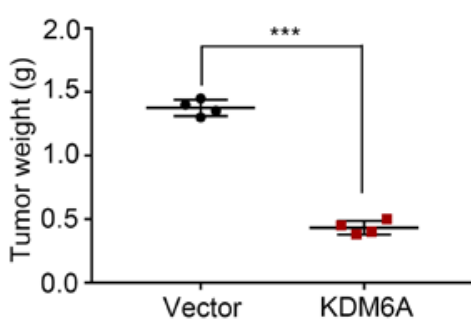

B

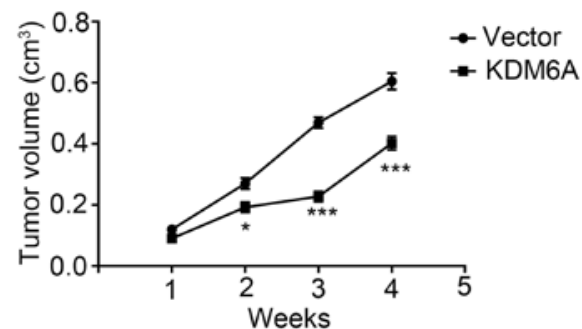

Vector

KDM6A
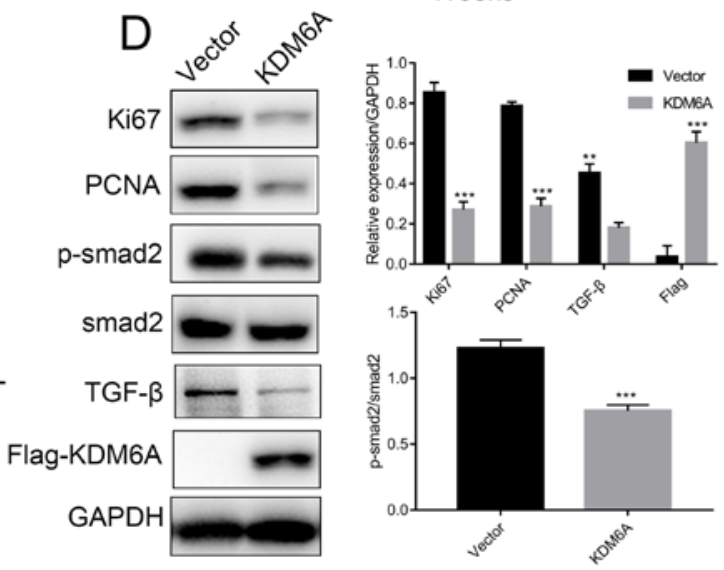

Figure 4. KDM6A overexpression suppresses hepatocellular carcinoma cell tumorigenesis in vivo. (A) Representative images of the tumors generated by empty vector- and KDM6A overexpression vector-transfected Huh7 cells. Tumor (B) volume and (C) weight. (D) The effect of KDM6A overexpression on the protein expression levels of TGF- $\beta$, p-smad2, Ki67 and PCNA in tumor samples. ${ }^{*} \mathrm{P}<0.05,{ }^{* *} \mathrm{P}<0.01$ and ${ }^{* * * *} \mathrm{P}<0.001$ vs. vector. KDM6A, lysine demethylase $6 \mathrm{~A}$; TGF- $\beta$, transforming growth factor- $\beta$; $p$, phosphorylated; PCNA, proliferating cell nuclear antigen; ns, not significant.

levels of KDM6A in 30 paired HCC and corresponding normal tissues were examined. KDM6A mRNA levels were significantly decreased in 25/30 (83\%) HCC samples compared with the corresponding normal samples (Fig. 1A). IHC analysis indicated that KDM6A was primarily expressed in the cytoplasm of both normal and tumor tissues. Consistent with the RT-qPCR results, HCC tissues displayed a decreased staining intensity compared with the corresponding normal tissues in two patients (Fig. 1B), which indicated that KDM6A expression was decreased in HCC tissues compared with corresponding normal tissues. In addition, the expression of KDM6A in several HCC cell lines was assessed. KDM6A expression was notably higher in YY-8103 and SNU-398 cells compared with Huh7 and LM3 cells (Fig. 1C and D).

KDM6A overexpression inhibits HCC cell proliferation in vitro. Based on the aforementioned clinical data, it was hypothesized that KDM6A may influence HCC cell proliferation in vitro. To evaluate the biological function of KDM6A in HCC cells, Huh7 and LM3 cell lines were transfected with empty (control) or KDM6A overexpression vectors. Western blotting was performed to confirm the transfection efficiency of KDM6A overexpression in HCC cells (Fig. 2A).

The MTT assay results indicated that the absorbance values of KDM6A-overexpression Huh7 and LM3 cells were significantly lower compared with empty vector-transfected cells on day 7 (Fig. 2B and C). In addition, the results of the crystal violet assay indicated that KDM6A overexpression markedly inhibited Huh7 and LM3 cell proliferation compared with empty vector-transfected cells (Fig. 2D). Similarly, the absorbance values of KDM6A-overexpression Huh7 and LM3 cells were significantly lower compared with empty vector-transfected cells (Fig. 2E).

KDM6A knockdown promotes HCC cell proliferation in vitro. Using gene-specific shRNAs, KDM6A expression was successfully knocked down in YY-8103 and SNU-398 cells (Fig. 3A). Similar to the overexpression experiments, the effect of KDM6A knockdown on HCC cell proliferation was assessed. The results of the MTT assay indicated that the absorbance values of KDM6A-knockdown YY-8103 and SNU-398 cells were significantly higher compared with SCR-transfected cells on day 7 (Fig. 3B and C). Consistently, the crystal violet assay results indicated that KDM6A knockdown markedly promoted YY-8103 and SNU-398 cell proliferation compared with SCR-transfected cells (Fig. 3D). Furthermore, the absorbance values of crystal violet in KDM6A-knockdown YY-8103 and SNU-398 cells were significantly higher compared with SCR-transfected cells (Fig. 3E).

KDM6A overexpression suppresses HCC cell tumorigenesis in vivo. Based on the in vitro results, empty vector- and KDM6A overexpression vector-transfected Huh7 cells were subcutaneously injected into the right flank of nude mice. Subsequently, the tumors were excised and photographed. Consistent with the in vitro results, KDM6A overexpression notably suppressed tumor growth compared with the empty vector control group in the xenograft mouse model (Fig. 4A). 

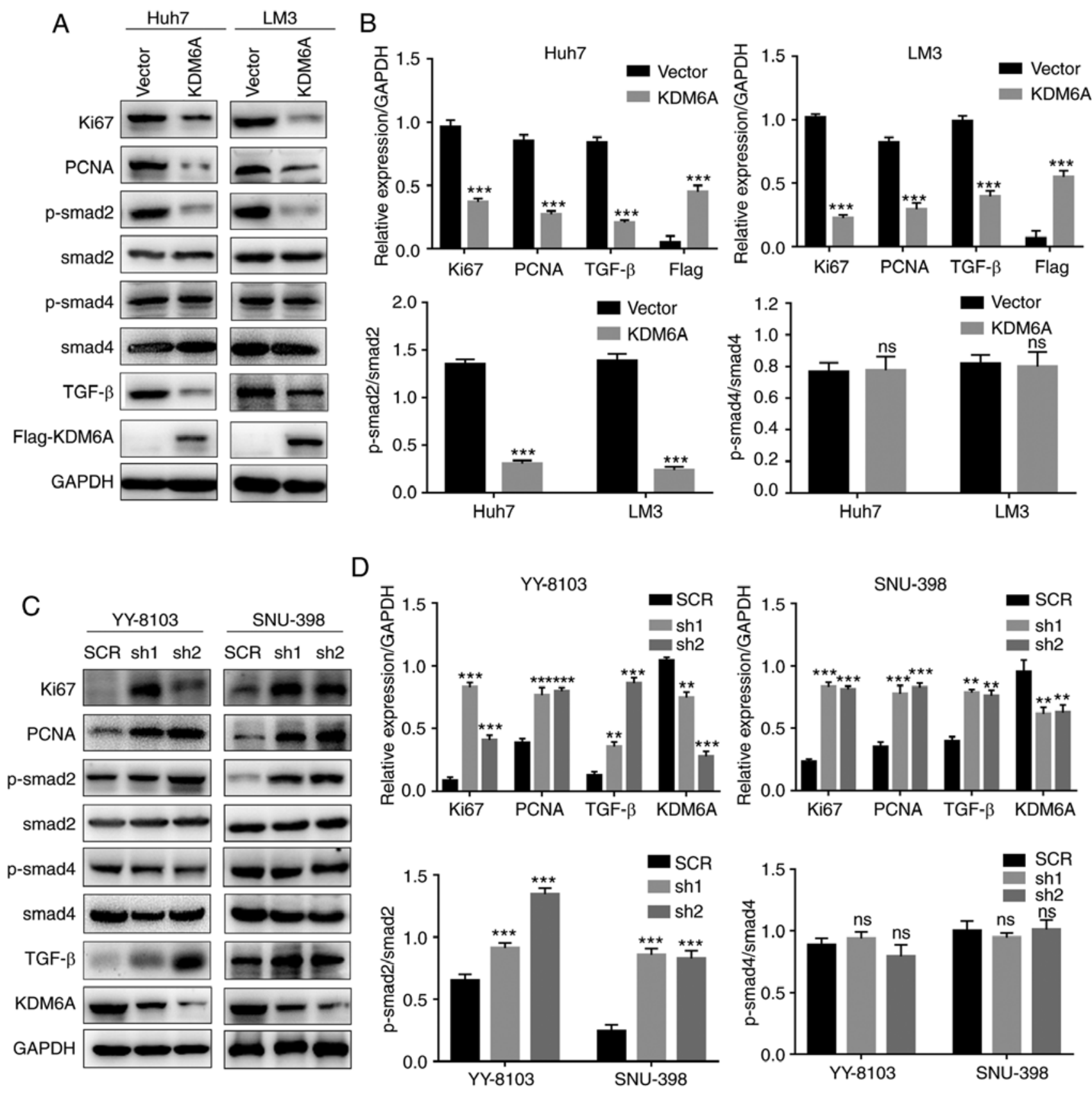

Figure 5. KDM6A negatively regulates the TGF- $\beta /$ SMAD signaling pathway. (A) The effect of KDM6A overexpression on the expression of TGF- $\beta$, p-smad2, p-smad4, Ki67 and PCNA in Huh7 and LM3 cells was (A) determined by western blotting and (B) semi-quantified. The effect of KDM6A knockdown on the expression of TGF- $\beta$, p-smad2, p-smad4, Ki67 and PCNA in YY-8103 and SNU-398 was (C) determined by western blotting and (D) semi-quantified. ${ }^{* *} \mathrm{P}<0.01$ and ${ }^{* * *} \mathrm{P}<0.001$ vs. vector or SCR. KDM6A, lysine demethylase $6 \mathrm{~A}$; TGF- $\beta$, transforming growth factor- $\beta ;$, phosphorylated; PCNA, proliferating cell nuclear antigen; SCR, scrambled; sh, short hairpin RNA; ns, not significant.

In particular, KDM6A overexpression tumors were lighter and grew at a reduced rate compared with empty vector control tumors $(\mathrm{P}<0.001$; Fig. $4 \mathrm{~B}$ and $\mathrm{C})$. The results suggested that KDM6A may serve as a tumor suppressor during HCC cell tumorigenesis in vivo. In addition, the western blotting results indicated that the protein expression levels of TGF- $\beta$, p-smad2, Ki67 and PCNA were significantly decreased in KDM6A overexpression tumors compared with empty vector control tumors (Fig. 4D).

KDM6A inhibits TGF- $\beta / S M A D$ signaling in HCC cells. To explore the molecular mechanism underlying the effects of KDM6A on HCC, p-smad2, total smad2, p-smad4, total smad 4 and TGF- $\beta$ protein expression levels were measured in KDM6A-overexpression and KDM6A-knockdown cells. The resultsindicated that theexpressionlevels of TGF- $\beta$ andp-Smad2 were significantly decreased in KDM6A-overexpression cells compared with empty vector control cells (Fig. 5A and B). By contrast, KDM6A-knockdown cells displayed significantly increased TGF- $\beta$ and $p$-smad 2 expression levels compared with SCR-transfected cells (Fig. 5C and D). However, there was no significant difference in the expression levels of p-smad4 in KDM6A-overexpression or KDM6A-knockdown cells compared with the corresponding control cells. In 
addition, KDM6A overexpression significantly decreased the expression levels of proliferation markers, such as Ki67 and PCNA, compared with empty vector control cells. By contrast, KDM6A knockdown significantly increased the expression levels of Ki67 and PCNA compared with SCR-transfected cells (Fig. 5).

\section{Discussion}

A number of inactivating somatic mutations and copy number losses related to KDM6A have been identified in numerous malignancies, including pancreatic ductal adenocarcinoma, multiple myeloma, and esophageal and urethral cancer (11). KDM6A gene loss induces squamous-like metastatic pancreatic cancer, specifically in women, by deregulating the COMPASS-like complex (15). Furthermore, loss of KDM6A has been correlated with a poor prognostic subtype of human pancreatic cancer, where it serves a functional role as a tumor suppressor (13). KDM6A can also inhibit the invasion of HCT-116 colon cancer cells by upregulating E-cadherin expression, whereas KDM6A knockdown enhances epithelial-mesenchymal transition (EMT)-mediated stem cell properties in breast cancer cells $(21,22)$. Moreover, Terashima et al (16) reported that KDM6A served an antagonistic role in TGF- $\beta$-induced lung cancer cell EMT and migration.

The present study indicated that KDM6A mRNA levels were significantly downregulated in HCC tissues compared with corresponding control tissues. Western blotting was performed to verify the transfection efficiency of KDM6A overexpression and knockdown in HCC cell lines. HCC cell proliferation was significantly inhibited by KDM6A overexpression compared with empty vector-transfected cells. Conversely, KDM6A knockdown promoted HCC cell proliferation compared with SCR-transfected cells. Furthermore, KDM6A overexpression also suppressed HCC cell tumorigenesis in vivo. Further mechanistic studies indicated that the expression levels of TGF- $\beta$ and p-smad 2 were significantly decreased by KDM6A overexpression compared with empty vector-transfected cells. By contrast, KDM6A knockdown increased TGF- $\beta$ and p-smad 2 expression levels, which are related to tumor progression (23), compared with SCR-transfected cells. Therefore, the present study indicated that KDM6A may inhibit HCC cell proliferation by negatively regulating TGF- $\beta$ /SMAD signaling. TGF- $\beta$ can influence gene expression by interacting with SMAD protein transcription factors, such as $\operatorname{smad} 2, \operatorname{smad} 3$ and $\operatorname{smad} 4$, to induce heterodimer formation $(24,25)$. TGF- $\beta$ signaling serves a complex role during the development and progression of various diseases (26). Specifically, activation of the TGF- $\beta$ signaling pathway has been associated with increased growth and invasion of malignant cells during the late stages of tumor progression (27-29). To the best of our knowledge, the present study was the first study to indicate that KDM6A may be involved in HCC cell proliferation.

In summary, the present study demonstrated that KDM6A may serve a critical role in HCC cell proliferation. The results provided a mechanistic understanding of the role of KDM6A during tumor development, suggesting that KDM6A may serve as a potential target for the diagnosis or treatment of HCC.

\section{Acknowledgements}

Not applicable.

\section{Funding}

No funding was received.

\section{Availability of data and materials}

The datasets used and/or analyzed during the current study are available from the corresponding author on reasonable request.

\section{Authors' contributions}

YL and JY conducted the experiments. XZ and HL provided the clinical samples. XZ, HL and JG analyzed the data. YL, XZ, HL and JG drafted and revised the manuscript. JG designed and supervised the study. All authors read and approved the final manuscript.

\section{Ethics approval and consent to participate}

The present study was approved by the Ethics Committee of the Gansu Provincial Hospital (approval no. KY-20190089; Lanzhou, China). Written informed consent was obtained from all subjects and the study was conducted in accordance with the Declaration of Helsinki.

\section{Patient consent for publication}

Not applicable.

\section{Competing interests}

The authors declare that they have no competing interests.

\section{References}

1. European Association for the Study of the Liver. Electronic address: easloffice@easloffice.eu; European Association for the Study of the Liver: EASL Clinical Practice Guidelines: Management of hepatocellular carcinoma. J Hepatol 69: 182-236, 2018.

2. Lu XJ, Shi Y, Chen JL and Ma S: Krüppel-like factors in hepatocellular carcinoma. Tumour Biol 36: 533-541, 2015.

3. Yu WB, Rao A, Vu V, Xu L, Rao JY and Wu JX: Management of centrally located hepatocellular carcinoma: Update 2016. World J Hepatol 9: 627-634, 2017.

4. Portolani N, Coniglio A, Ghidoni S, Giovanelli M, Benetti A, Tiberio GA and Giulini SM: Early and late recurrence after liver resection for hepatocellular carcinoma: Prognostic and therapeutic implications. Ann Surgery 243: 229-235, 2006.

5. Hu J and Gao DZ: Distinction immune genes of hepatitis-induced heptatocellular carcinoma. Bioinformatics 28: 3191-3194, 2012.

6. Marquardt JU, Galle PR and Teufel A: Molecular diagnosis and therapy of hepatocellular carcinoma (HCC): An emerging field for advanced technologies. J Hepatol 56: 267-275, 2012.

7. Hong S, Cho YW, Yu LR, Yu H, Veenstra TD and Ge K: Identification of JmjC domain-containing UTX and JMJD3 as histone $\mathrm{H} 3$ lysine 27 demethylases. Proc Natl Acad Sci USA 104: 18439-18444, 2007.

8. Lee MG, Villa R, Trojer P, Norman J, Yan KP, Reinberg D, Di Croce L and Shiekhattar R: Demethylation of H3K27 regulates polycomb recruitment and $\mathrm{H} 2 \mathrm{~A}$ ubiquitination. Science 318: 447-450, 2007. 
9. Jiang W, Wang J and Zhang Y: Histone H3K27me3 demethylases KDM6A and KDM6B modulate definitive endoderm differentiation from human ESCs by regulating WNT signaling pathway. Cell Res 23: 122-130, 2013.

10. Schuettengruber B, Bourbon HM, Di Croce L and Cavalli G: Genome Regulation by Polycomb and Trithorax: 70 Years and Counting. Cell 171: 34-57, 2017.

11. van Haaften G, Dalgliesh GL, Davies H, Chen L, Bignell G, Greenman C, Edkins S, Hardy C, O'Meara S, Teague J, et al: Somatic mutations of the histone H3K27 demethylase gene UTX in human cancer. Nat Genet 41: 521-523, 2009.

12. Terashima M, Ishimura A, Yoshida M, Suzuki Y, Sugano S and Suzuki T: The tumor suppressor $\mathrm{Rb}$ and its related $\mathrm{Rbl} 2$ genes are regulated by Utx histone demethylase. Biochem Biophys Res Commun 399: 238-244, 2010.

13. Watanabe S, Shimada S, Akiyama Y, Ishikawa Y, Ogura T, Ogawa K, Ono H, Mitsunori Y, Ban D, Kudo A, et al: Loss of KDM6A characterizes a poor prognostic subtype of human pancreatic cancer and potentiates HDAC inhibitor lethality. Int J Cancer 145: 192-205, 2019.

14. Ler LD, Ghosh S, Chai X, Thike AA, Heng HL, Siew EY, Dey S, Koh LK, Lim JQ, Lim WK, et al: Loss of tumor suppressor KDM6A amplifies PRC2-regulated transcriptional repression in bladder cancer and can be targeted through inhibition of EZH2. Sci Transl Med 9: eaai8312, 2017.

15. Andricovich J, Perkail S, Kai Y, Casasanta N, Peng W and Tzatsos A: Loss of KDM6A activates super-enhancers to induce gender-specific squamous-like pancreatic cancer and confers sensitivity to BET inhibitors. Cancer Cell 33: 512-526.e8, 2018.

16. Terashima $M$, Ishimura $A$, Wanna-Udom $S$ and Suzuki T: Epigenetic regulation of epithelial-mesenchymal transition by KDM6A histone demethylase in lung cancer cells. Biochem Biophys Res Commun 490: 1407-1413, 2017.

17. Wang Y, Liu DP, Chen PP, Koeffler HP, Tong XJ and Xie D: Involvement of IFN regulatory factor (IRF)-1 and IRF-2 in the formation and progression of human esophageal cancers. Cancer Res 67: 2535-2543, 2007.

18. Qiu Z, Zou K, Zhuang L, Qin J, Li H, Li C, Zhang Z, Chen X, Cen J, Meng Z, et al: Hepatocellular carcinoma cell lines retain the genomic and transcriptomic landscapes of primary human cancers. Sci Rep 6: 27411, 2016.
19. Vatandoost J and Kafi Sani K: A study of recombinant factor IX in Drosophila insect $\mathrm{S} 2$ cell lines through transient gene expression technology. Avicenna J Med Biotechnol 10: 265-268, 2018.

20. Sikes RS: 2016 Guidelines of the American Society of Mammalogists for the use of wild mammals in research and education. J Mammal 97: 663-688, 2016.

21. Zha L, Cao Q, Cui X, Li F, Liang H, Xue B and Shi H: Epigenetic regulation of E-cadherin expression by the histone demethylase UTX in colon cancer cells. Med Oncol 33: 21, 2016.

22. Choi HJ, Park JH, Park M, Won HY, Joo HS, Lee CH, Lee JY and Kong G: UTX inhibits EMT-induced breast CSC properties by epigenetic repression of EMT genes in cooperation with LSD1 and HDAC1. EMBO Rep 16: 1288-1298, 2015.

23. Dai J, Xu M, Zhang X, Niu Q, Hu Y, Li Y and Li S: Bi-directional regulation of TGF- $\beta /$ Smad pathway by arsenic: A systemic review and meta-analysis of in vivo and in vitro studies. Life Sci 220: 92-105, 2019.

24. Derynck R and Zhang YE: Smad-dependent and Smad-independent pathways in TGF-beta family signalling. Nature 425: 577-584, 2003.

25. Wrana JL, Attisano L, Wieser R, Ventura F and Massagué J: Mechanism of activation of the TGF-beta receptor. Nature 370 341-347, 1994.

26. Moses HL, Roberts AB and Derynck R: The discovery and early days of TGF- $\beta$ : A historical perspective. Cold Spring Harb Perspect Biol 8: a021865, 2016.

27. Derynck R, Akhurst RJ and Balmain A: TGF-beta signaling in tumor suppression and cancer progression. Nat Genet 29: 117-129, 2001.

28. Pasche B: Role of transforming growth factor beta in cancer. J Cell Physiol 186: 153-168, 2001.

29. Elliott RL and Blobe GC: Role of transforming growth factor Beta in human cancer. J Clin Oncol 23: 2078-2093, 2005.

This work is licensed under a Creative Commons Attribution-NonCommercial-NoDerivatives 4.0 International (CC BY-NC-ND 4.0) License. 